\title{
Emerging multi-modal diagnostic approaches for moyamoya disease
}

\author{
Jae W. Song', Zhaoyang Fan ${ }^{2}$ \\ ${ }^{1}$ Department of Radiology, Hospital of the University of Pennsylvania, Philadelphia, PA, USA; ${ }^{2}$ Biomedical Imaging Research Institute, Cedars-Sinai \\ Medical Center, Los Angeles, CA, USA \\ Correspondence to: Zhaoyang Fan, PhD. Biomedical Imaging Research Institute, Cedars-Sinai Medical Center, 8700 Beverly Blvd, Pacific Theatres \\ 400, Los Angeles, CA 90048, USA. Email: Zhaoyang.Fan@cshs.org. \\ Comment on: Ya J, Zhou D, Ding J, et al. High-resolution combined arterial spin labeling MR for identifying cerebral arterial stenosis induced by \\ moyamoya disease or atherosclerosis. Ann Transl Med 2020;8:87.
}

Submitted May 24, 2020. Accepted for publication Jul 13, 2020.

doi: $10.21037 /$ atm-20-4215

View this article at: http://dx.doi.org/10.21037/atm-20-4215

Moyamoya disease (MMD) is a steno-occlusive vasculopathy most commonly affecting the intracranial internal carotid artery terminus. It can lead to hypoperfusion and ischemia due to reduced blood flow in the major vessels of the anterior circulation. Compensatory development of basal, cortical, and leptomeningeal collateral vessels is associated with MMD. Despite compensatory collateralization, up to two thirds of patients with MMD have symptomatic progression over a 5 -year period and outcomes are poor without treatment $(1,2)$. Clinical outcome is associated with the neurologic status at the time of treatment (3) and given a $2.6 \%$ rate of symptomatic progression after surgical revascularization (4), early diagnosis and therapy are critical for a better prognosis.

Angiographic appearances of MMD can be similar to stenosis related to other vasculopathies, such as intracranial atherosclerosis (ICAS), and pose a diagnostic challenge. However, because treatment strategies and prognoses differ between ICAS and MMD, it is important to distinguish these two entities. Multimodal approaches using both structural and physiologic data may be of added value to diagnose and support management strategies.

Ya and colleagues investigated the use of combined highresolution intracranial vessel wall MR imaging (VWI) and pseudo-continuous arterial spin labeling (pCASL) techniques to determine differences in morphologic and perfusional characteristics of ICAS and MMD (5). VWI is a black blood MR imaging technique that has substantially been optimized over the past decade for the intracranial vessels (6). 3D turbo spin-echo with variable refocusing flip angles, which was also used in their work, has become a method of choice for non-invasive assessment of the intracranial vessel wall (7). Compared to conventional vessel imaging techniques that detect changes in lumen diameter, VWI shows changes in the vessel wall itself. pCASL is an MR technique that measures cerebral blood flow (CBF), an important physiologic parameter commonly altered in many cerebrovascular conditions. It is considered the best ASL approach given its higher SNR and tagging efficiency compared to continuous ASL and pulsed ASL, respectively (8).

Using both advanced imaging techniques, the authors compared structural and perfusional changes in 20 subjects with ICAS and 20 subjects diagnosed with various stages of MMD. The results showed significantly smaller outer and inner wall diameters and maximum wall thickness, higher mean $\mathrm{CBF}$, cerebral blood volume (CBV), and arterial transit time (ATT), and higher maximum $\mathrm{CBF}$ and $\mathrm{CBV}$ values in patients diagnosed with MMD than in ICAS. Furthermore, on visual inspection, 14 of 20 MMD patients (Suzuki stages II-V) showed hyper-perfusion spots on the CBF maps. Hyper-perfusion spots were not identified in 6 MMD (Suzuki stage VI) patients nor in the 20 patients diagnosed with ICAS. Based on these results, the authors concluded a multi-modal approach using both VWI and pCASL-MR may help distinguish between these two entities.

The results suggest a promising multi-modal diagnostic approach for evaluating MMD versus ICAS. The VWI 
results are concordant with several other studies showing smaller vessel wall outer and inner wall diameters (9-11). In MMD, the affected arteries reportedly undergo constrictive (inward) remodeling and histopathologic studies also support the finding of reduced outer vessel diameters, thinner media and thicker intima in arteries affected by MMD (12). This remodeling pattern is in contrast to ICAS, which typically has an inflammatory pathogenesis and is associated with positive (outward) remodeling to accommodate plaque development (13). Multi-contrast VWI has also been used to help distinguish among stenoocclusive diseases, including MMD (14). Patterns of eccentric post-contrast enhancement and heterogeneous $\mathrm{T} 2$-weighted signal intensity are suggested to increase diagnostic confidence for identifying ICAS (14). Using a multi-contrast VWI protocol to evaluate for additional VWI-based features of wall pathology may be a future consideration.

The results from imaging with pCASL-MR also show interesting significant perfusional differences between MMD and ICAS. ASL has been previously used to identify the presence and intensity of collateral perfusion in MMD (15). While both diseases show hypo-perfusion in the territories of the stenotic arteries, the authors also highlight the presence of hyper-perfusion spots in $70 \%$ of the MMD cases. No hyper-perfusion spots were identified in the ICAS cases. Among the MMD cases, hyper-perfusion foci were identified only in MMD stages II to V. The absence of hyperperfusion foci in MMD stages I and VI could be explained by the classification criteria of the Suzuki staging system where intracranial collaterals are absent in these very early and advanced stages (16). Thus the authors suggest the hyper-perfusion foci may be associated with moyamoya collateral compensation or microaneurysms of collateral vessels. Future work with a larger sample size and distribution of subjects of various MMD stages would be of value to better understand how the degree of collateralization affects the results.

Many different imaging modalities have been investigated to differentiate MMD from other stenoocclusive vasculopathies. The combination of two noninvasive imaging modalities that assess both structural and physiologic changes have strong potential as a diagnostic approach. From these exams, both quantitative and qualitative multiparametric data could further improve diagnostic specificity. Here, the authors address this combined approach and show early promise using this approach to increase diagnostic confidence and accuracy to distinguish MMD from ICAS.

\section{Acknowledgments}

Funding: ZF received salary support from National Institutes of Health (NIH/NHLBI R01 HL147355).

\section{Footnote}

Provenance and Peer Review: This article was commissioned by the editorial office, Annals of Translational Medicine. The article did not undergo external peer review.

Conflicts of Interest: Both authors have completed the ICMJE uniform disclosure form (available at http://dx.doi. org/10.21037/atm-20-4215). Both authors have no conflicts of interests to declare.

Ethical Statement: The authors are accountable for all aspects of the work in ensuring that questions related to the accuracy or integrity of any part of the work are appropriately investigated and resolved.

Open Access Statement: This is an Open Access article distributed in accordance with the Creative Commons Attribution-NonCommercial-NoDerivs 4.0 International License (CC BY-NC-ND 4.0), which permits the noncommercial replication and distribution of the article with the strict proviso that no changes or edits are made and the original work is properly cited (including links to both the formal publication through the relevant DOI and the license). See: https://creativecommons.org/licenses/by-nc-nd/4.0/.

\section{References}

1. Choi JU, Kim DS, Kim EY, et al. Natural history of moyamoya disease: comparison of activity of daily living in surgery and non surgery groups. Clin Neurol Neurosurg 1997;99 Suppl 2:S11-S18.

2. Kuroda S, Ishikawa T, Houkin K, et al. Incidence and clinical features of disease progression in adult moyamoya disease. Stroke 2005;36:2148-53.

3. Scott RM, Smith JL, Robertson RL, et al. Long-term outcome in children with moyamoya syndrome after cranial revascularization by pial synangiosis. J Neurosurg 2004;100:142-9.

4. Fung LW, Thompson D, Ganesan V. Revascularisation surgery for paediatric moyamoya: a review of the literature. 
Childs Nerv Syst 2005;21:358-64.

5. Ya J, Zhou D, Ding J, et al. High-resolution combined arterial spin labeling MR for identifying cerebral arterial stenosis induced by moyamoya disease or atherosclerosis. Ann Transl Med 2020;8:87.

6. Song JW, Moon BF, Burke MP, et al. MR Intracranial Vessel Wall Imaging: A Systematic Review. J Neuroimaging 2020;30:428-42.

7. Mandell DM, Mossa-Basha M, Qiao Y, et al. Intracranial Vessel Wall MRI: Principles and Expert Consensus Recommendations of the American Society of Neuroradiology. AJNR Am J Neuroradiol 2017;38:218-29.

8. Alsop DC, Detre JA, Golay X, et al. Recommended implementation of arterial spin-labeled perfusion MRI for clinical applications: A consensus of the ISMRM perfusion study group and the European consortium for ASL in dementia. Magn Reson Med 2015;73:102-6.

9. Kaku Y, Morioka M, Ohmori Y, et al. Outer-diameter narrowing of the internal carotid and middle cerebral arteries in moyamoya disease detected on 3D constructive interference in steady-state MR image: is arterial constrictive remodeling a major pathogenesis?. Acta Neurochir (Wien) 2012;154:2151-7.

Cite this article as: Song JW, Fan Z. Emerging multi-modal diagnostic approaches for moyamoya disease. Ann Transl Med 2020;8(19):1208. doi: 10.21037/atm-20-4215
10. Ryoo S, Cha J, Kim SJ, et al. High-resolution magnetic resonance wall imaging findings of Moyamoya disease. Stroke 2014;45:2457-60.

11. Yuan M, Liu ZQ, Wang ZQ, et al. High-resolution MR imaging of the arterial wall in moyamoya disease. Neurosci Lett. 2015;584:77-82.

12. Takagi Y, Kikuta K, Nozaki K, et al. Histological features of middle cerebral arteries from patients treated for Moyamoya disease. Neurol Med Chir (Tokyo) 2007;47:1-4.

13. Qiao Y, Anwar Z, Intrapiromkul J, et al. Patterns and Implications of Intracranial Arterial Remodeling in Stroke Patients. Stroke 2016;47:434-40.

14. Mossa-Basha M, de Havenon A, Becker KJ, et al. Added Value of Vessel Wall Magnetic Resonance Imaging in the Differentiation of Moyamoya Vasculopathies in a NonAsian Cohort. Stroke 2016;47:1782-8.

15. Zaharchuk G, Do HM, Marks MP, et al. Arterial spinlabeling MRI can identify the presence and intensity of collateral perfusion in patients with moyamoya disease. Stroke 2011;42:2485-91.

16. Suzuki J, Kodama N. Moyamoya disease--a review. Stroke $1983 ; 14: 104-9$. 